\title{
Description Lookup based UPnP Extension for Wireless Sensor Networks
}

\author{
Jongwoo Sung, Seong Hoon Kim, Young-Joo Kim, Daeyoung Kim \\ Korea Advanced Institute of Science and Technology \\ \{jwsung, shkim1908, yjkim73, kimd\}@kaist.ac.kr
}

\begin{abstract}
Established service discovery protocols such as $U P n P$ allow control points to find devices and services and to retrieve descriptions about them in order to learn all about the device and services. However, general device-control point approaches are not suitable for wireless sensor networks due to severe resource constraints and gateway architecture. To alleviate the problems we change discovery processes: 1) sensor node announces uniquely assigned identifiers; 2) base station retrieves additional descriptions for sensor nodes using lookup facilities; and 3) control points communicate with the base station to browse a list of descriptions and to send control commands to sensor nodes via standard UPnP protocols. In this paper we introduce our description lookup-based UPnP extension system for wireless sensor networks.
\end{abstract}

Index Terms - service discovery, description, lookup, UPnP, wireless sensor networks

\section{Introduction}

Established service discovery protocols such as UPnP allows control points to find devices and services and to retrieve descriptions about them in order to learn all about the device and services. The description is expressed in XML and includes a list of commands, arguments for actions and state variables as well as manufacturer specific information.

To use service discovery protocols in wireless sensor networks gateway architecture that plays a role of translating messages and protocols between UPnP control points and non-UPnP sensor nodes has been widely adopted. Specifically, an intermediate base station act as a single UPnP device that provides services on behalf all sensor nodes [1] or it creates virtual UPnP devices for non-UPnP sensor nodes based on ZigBee profiles [2][3].

Although simple service discovery protocol (SSDP) allows devices to advertise their services using limited

This work was supported by Korea Science \& Engineering

Foundation through the national research lab (NRL) Program 2009. values (service type, identifier, and a pointer to more detailed information) control points need to retrieve sensor node descriptions to access them. However, storing XML based descriptions in tiny sensor nodes and transferring them to base stations or control points are not suitable for wireless sensor networks due to severe resource constraints such as limited memory space and energy consumptions during communications. Changing sensor node profiles into virtual UPnP devices raises a strong dependency between sensor nodes and the base station. In addition, large numbers of virtual devices [2][3] easily create many UPnP broadcasting messages.

To alleviate the problems we change discovery processes: 1) devices include unique identifiers in announcement messages; 2) base stations retrieve standard UPnP descriptions for sensor nodes using lookup facilities and the base station changes URLs to control in the descriptions with a local IP address; and 3) control points communicate with the base stations to get descriptions or to invoke service objects (services offered by sensor nodes). The base station translates UPnP messages into message formats for sensor nodes, and vice versa using conversion rules in the service objects. Figure 1 shows a logical diagram.

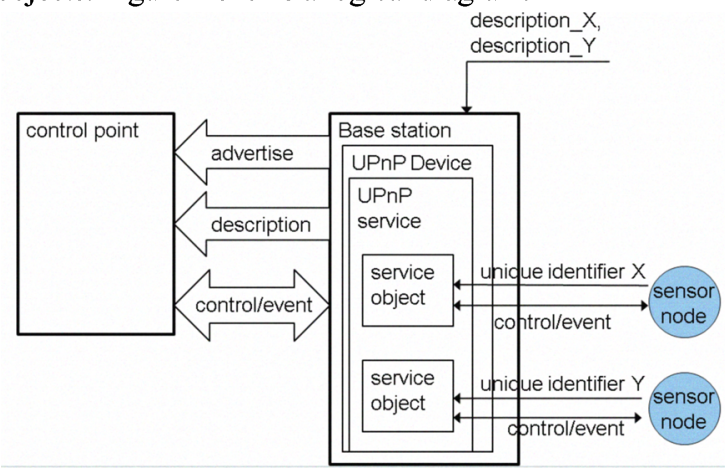

Figure 1 description lookup based UPnP logical diagram

\section{Proposed Architecture}

To enable the description lookup based UPnP 
extension for wireless sensor networks lookup facilities and UPnP extensions in wireless sensor networks are explained.

\section{A. lookup architecture}

UPnP descriptions for all sensor nodes are automatically retrieved from Internet servers as we do with software drivers. Lookup facilities consist of unique identifiers, distributed profile servers, and resolving system [4]. Overall architecture is shown in Figure 2. Unique identifiers that are used as keys to lookup location of descriptions are assigned to each sensor node. Descriptions are stored in Internet hosts, called profile servers, instead of sensor node memory. The profile servers provide web interfaces for the base station to search or download descriptions using the identifier of a desired sensor node.

To find authorized profile servers having descriptions for desired sensor nodes DNS based resolving system that changes identifiers to addresses is adopted. The base station acts as a gateway between UPnP networks and sensor networks, and creates service objects (service offered by sensor nodes) for retrieved descriptions.

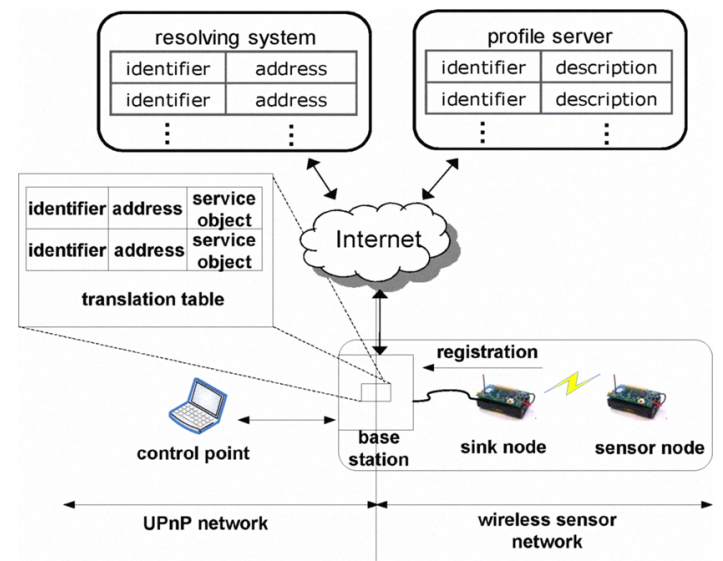

Figure 2 description lookup based UPnP architecture

$B$. Sensor network operations

To access sensor networks in a plug and play manner in Figure 2, new sensor nodes need to be identified by control points. Sensor nodes announce their unique identifiers to a base station whenever they have any topology change. Registration message includes a sensor node identifier and a dynamically assigned network address. The base station maintains a list of identifications and network addresses mapping, called translation table. The table is continuously up-to-dated by following update message that is issued whenever sensor nodes have any change (such as leaving) or update time, set by users, is expired.

Descriptions retrieved from servers are standard XML documents for UPnP devices and they include conversion rules for communicating with sensor nodes. Although further translation of the description is not necessary, the base station needs to change URLs to control in description with a local IP address of it.

Retrieved sensor node descriptions called service objects are stored in the base station so that control points can query them and invoke sensor node services using standard UPnP operations. The base station translates UPnP messages into message formats for sensor nodes, and vice versa using conversion rules in service objects.

Because descriptions are queried by the control point via standard UPnP operation, simple service discovery protocol (SSDP) message is suppressed to prevent broadcast storms caused by multiple announcement messages from large numbers of sensor nodes.

\section{Conclusion}

We proposed description lookup based UPnP extension for wireless sensor networks. With lookup facilities base stations retrieve descriptions from the authorized profile servers. We remove the dependency between the base station and sensor nodes as well as overhead from handling XML based descriptions in tiny sensor nodes. To remove broadcast storms caused by large numbers of announcements from virtual devices, service descriptions are transferred to the control point via UPnP controls. Precise evaluation of proposed architecture against varying numbers of sensor nodes will be our future work.

\section{References}

[1] Hyungjoo Song, Daeyoung Kim, Kangwoo Lee, Jongwoo Sung, "UPnP-based Sensor Network Managemetn Architecture and Implementation," Second International Conference on Mobile Computing and Ubiquitous Networking (ICMU 2005), Osaka University, Osaka, JAPAN, April 2005.

[2] "DLNA/UPnP-ZigBee Gateway Specifications," ALPHA SYSTEMS INC. and interoperability Tecnonology Association for Infomraiton Processing, Japan, August 2007.

[3] Seong Hoon Kim, Jeong Seok Kang, Kwang Kook Lee, Hong Seong Park, Sung Ho Baeg, and Jea Han Park, "A UPnPZigBee Software Bridge," 2007 International Conference on Computational Science and its Applications, LNCS, vol 4705, pp. 346-359, Aug. 2007.

[4] Jongwoo Sung, Youngsoo Kim, Taehong Kim, Young-Joo Kim, Daeyoung Kim, "Internet Metadata Framework for Plug and Play Wireless Sensor Networks", IEEE Sensors Application Symposium (SAS), New Orleans, Louisiana, USA, 17-19 February 2009. 